ANNALES

POLONICI MATHEMATICI

$86.1(2005)$

\title{
A new approach to the existence results for orientor fields with Nicoletti's boundary conditions
}

\author{
by StanisŁaw Domachowski (Gdańsk)
}

\begin{abstract}
Applying a global bifurcation theorem for convex-valued completely continuous mappings we prove some existence theorems for convex-valued differential inclusions of the form $x^{\prime} \in F(t, x)$, where $x$ satisfies the Nicoletti boundary conditions.
\end{abstract}

1. Introduction. In this paper we consider the Nicoletti boundary value problem for the first order differential inclusions

$$
\left\{\begin{array}{l}
x^{\prime}(t) \in F(t, x(t)) \quad \text { for a.e. } t \in(a, b), \\
l(x)=0
\end{array}\right.
$$

where $F:[a, b] \times \mathbb{R}^{k} \rightarrow \mathrm{cf}\left(\mathbb{R}^{k}\right)$ is a convex-valued mapping and $l: C\left([a, b], \mathbb{R}^{k}\right)$ $\rightarrow \mathbb{R}^{k}$ is given by

$$
l\left(x_{1}, \ldots, x_{k}\right)=\left(x_{1}(a), x_{2}(b), x_{3}\left(t_{3}\right), \ldots, x_{k}\left(t_{k}\right)\right) \text {, where } t_{3}, \ldots, t_{k} \in[a, b] .
$$

The Nicoletti single-valued and multi-valued boundary value problem has been considered by several authors (see for instance [5], [6], [10]). In these papers it is assumed that the mapping $F$ satisfies the Carathéodory conditions and the inequality

$$
|F(t, x)| \leq p(t)|x|+q(t) \quad \text { for } x \in \mathbb{R}^{k} \text { and } t \in[a, b],
$$

where $p, q:[a, b] \rightarrow \mathbb{R}_{+}$are integrable functions, and $p$ satisfies the inequality

$$
\int_{a}^{b} p(t) d t<\frac{\pi}{2} .
$$

Lasota and Olech [6] considered the single-valued Nicoletti problem. They showed that for $F$ satisfying (1.2) the condition (1.3) is the best possible sufficient condition for the existence of solution of (1.1).

2000 Mathematics Subject Classification: Primary 47H04; Secondary 34A60.

Key words and phrases: differential inclusion, global bifurcation theorem, Nicoletti boundary conditions. 
Our assumptions refer to the behaviour of $F(t, x)$ for $|x|$ close to 0 and to $\infty$. The main tool we use is a global bifurcation theorem for convexvalued completely continuous mappings. In Section 2 we state the main existence theorems. In Section 3 we give auxiliary lemmas, and transform the Nicoletti boundary value problem to appropriate second order boundary value problems. Finally, in Section 4 we prove the existence theorems.

2. Main theorems. Let $E$ be a real Banach space. We denote by $\operatorname{cf}(E)$ the family of all non-empty, closed, bounded and convex subsets of $E$, and by $\mathrm{D}(A, B)$ the Hausdorff distance between $A, B \in \operatorname{cf}(E)$. In particular we put $|A|=\mathrm{D}(A,\{0\})$.

Let $X$ be a closed non-empty subset of $E$. A multi-valued mapping $\Phi$ : $X \rightarrow \operatorname{cf}(E)$ is called upper semicontinuous (u.s.c.) if for each open set $U \subset E$ the set $\{x \in X: \Phi(x) \subset U\}$ is open in $X$.

Let $I \subset \mathbb{R}$ be a closed interval. A multi-valued mapping $\Phi: I \rightarrow \operatorname{cf}\left(\mathbb{R}^{k}\right)$ is called measurable if for every open set $U \subset \mathbb{R}^{k}$ the set $\{x \in I: \Phi(x) \cap U \neq \emptyset\}$ is Lebesgue measurable.

For $x=\left(x_{1}, \ldots, x_{k}\right) \in \mathbb{R}^{k}$ we write $|x|=\left(\sum_{i=1}^{k} x_{i}^{2}\right)^{1 / 2}$, and let $\Pi_{i}: \mathbb{R}^{k} \rightarrow$ $\mathbb{R}$ be the linear projection given by $\Pi_{i}\left(x_{1}, \ldots, x_{k}\right)=x_{i}$ for $i=1, \ldots, k$.

Recall that the multi-valued mapping $F:[a, b] \times \mathbb{R}^{k} \rightarrow \operatorname{cf}\left(\mathbb{R}^{k}\right)$ satisfies the Carathéodory conditions if:

(i) for each $x \in \mathbb{R}^{k}$ the mapping $F(\cdot, x)$ is measurable;

(ii) for each $t \in[a, b]$ the mapping $F(t, \cdot)$ is u.s.c.;

(iii) for each $R>0$ there exists a function $m_{R} \in L^{1}(a, b)$ such that for each $x \in \mathbb{R}^{k}$ with $|x| \leq R$ we have $|F(t, x)| \leq m_{R}(t)$ a.e. on $[a, b]$.

Theorem 1. Assume that $F:[a, b] \times \mathbb{R}^{k} \rightarrow \operatorname{cf}\left(\mathbb{R}^{k}\right)$ satisfies the Carathéodory conditions and

(2.1) there exists $\delta>0$ and an integrable function $\psi:[a, b] \rightarrow \mathbb{R}_{+}$such that

$$
|F(t, x)| \leq \psi(t)|x| \quad \text { for } t \in[a, b],|x| \leq \delta \quad \text { and } \quad \int_{a}^{b} \psi(t) d t<\frac{\pi}{2} ;
$$

(2.2) for every $\varepsilon>0$ there exists $R_{0}>0$ such that

$$
\mathrm{D}\left(\left(\Pi_{1} \circ F\right)(t, x),\left\{x_{2}\right\}\right)+\mathrm{D}\left(\left(\Pi_{2} \circ F\right)(t, x),\left\{-M\left|x_{1}\right|\right\}\right) \leq \varepsilon|x|
$$

for $t \in[a, b],\left|x_{1}\right|+\left|x_{2}\right| \geq R_{0}$ and $M>(\pi / 2(b-a))^{2}$;

(2.3) there exists $R_{1}>0$ and integrable functions $\psi_{i}:[a, b] \rightarrow \mathbb{R}_{+}$with

$$
\left|\left(\Pi_{i} \circ F\right)(t, x)\right| \leq \psi_{i}(t)\left|x_{i}\right| \quad \text { for } t \in[a, b],|x| \geq R_{1} \text { and } i=3, \ldots, k \text {. }
$$

Then there exists a non-trivial solution of the boundary value problem (1.1).

Theorem 2. Assume that $F:[a, b] \times \mathbb{R}^{k} \rightarrow \mathrm{cf}\left(\mathbb{R}^{k}\right)$ satisfies the Carathéodory conditions and conditions (2.1), (2.2), and 
(2.4) there exists $R_{1}>0$ and an integrable function $\psi:[a, b] \rightarrow \mathbb{R}_{+}$such that

$$
\begin{aligned}
& \left(\sum_{i=3}^{k}\left|\left(\Pi_{i} \circ F\right)(t, x)\right|^{2}\right)^{1 / 2} \leq \psi(t)\left(\sum_{i=3}^{k} x_{i}^{2}\right)^{1 / 2} \quad \text { for } t \in[a, b],|x| \geq R_{1} \\
& \text { and } \int_{a}^{b} \psi(t) d t<\pi / 2 .
\end{aligned}
$$

Then there exists a non-trivial solution of the boundary value problem (1.1).

3. Auxiliary lemmas. Let $\psi:[a, b] \rightarrow \mathbb{R}_{+}$satisfy (2.1). Then there exists $\alpha>1$ such that $\alpha \int_{a}^{b} \psi(t) d t<\pi / 2$. Let $q_{1}, q_{2}:(0, \infty) \rightarrow[0,1]$ be a continuous partition of unity subordinate to the open cover $\{(0, \alpha),(\beta, \infty)\}$ of the interval $(0, \infty)$, where $1<\beta<\alpha$, and let $p: \mathbb{R}^{k} \rightarrow \mathbb{R}^{k}$ be given by $p\left(x_{1}, x_{2}, \ldots, x_{k}\right)=\left(-x_{2}, M\left|x_{1}\right|, 0, \ldots, 0\right)$.

We start with the following scalar boundary value problem:

$$
\left\{\begin{array}{l}
x^{\prime \prime}(t)+\lambda x(t)=0 \\
x(a)=x^{\prime}(b)=0
\end{array}\right.
$$

It is well known (cf. [4]) that there exists exactly one eigenvalue $\mu_{0}=$ $(\pi / 2(b-a))^{2}$ of $(3.1)$ for which there exists an eigenvector $x_{0}:[a, b] \rightarrow \mathbb{R}$ such that $x_{0}(t)>0$ for $t \in(a, b)$.

The following fact is a consequence of the properties of Green's function (cf. [2]-[4]).

If $\mu>(\pi / 2(b-a))^{2}, \eta>0$ and $x_{0}:[a, b] \rightarrow \mathbb{R}$ is as above then the scalar problem

$$
\left\{\begin{array}{l}
x^{\prime \prime}(t)+\mu x(t)+\eta x_{0}(t)=0 \\
x(a)=x^{\prime}(b)=0 \\
x(t) \geq 0
\end{array}\right.
$$

has no solutions.

Let $x^{0}:[a, b] \rightarrow \mathbb{R}^{k}$ be given by $x^{0}(t)=\left(0, x_{0}(t), 0, \ldots, 0\right)$. We now associate with the problem (1.1) the following two-parameter family of boundary value problems:

$\left(2_{(\lambda, \tau)}\right)\left\{\begin{array}{lr}x^{\prime}(t) \in \lambda \tau q_{1}(\lambda) F(t, x(t))-\lambda q_{2}(\lambda) p(x(t))-(1-\tau) q_{2}(\lambda) x^{0}(t) \\ l(x)=0, & \text { for a.e. } t \in(a, b),\end{array}\right.$

for $\lambda \in(0, \infty)$ and $\tau \in[0,1]$. An absolutely continuous function $x:[a, b] \rightarrow$ $\mathbb{R}^{k}$ satisfying $\left(2_{(\lambda, \tau)}\right)$ is called a solution of $\left(2_{(\lambda, \tau)}\right)$. For every pair $(\lambda, \tau) \in$ $(0, \infty) \times[0,1]$ denote by $S_{(\lambda, \tau)}$ the set of all solutions of the problem $\left(2_{(\lambda, \tau)}\right)$. In what follows we will make use of the following fact. 
(3.3) (Lasota-Olech [5]) Let $p:[a, b] \rightarrow \mathbb{R}_{+}$be an integrable function with $\int_{a}^{b} p(t) d t<\pi / 2$, let $t_{1}, \ldots, t_{k} \in[a, b]$ and let $x=\left(x_{1}, \ldots, x_{k}\right):$ $[a, b] \rightarrow \mathbb{R}^{k}$ be an absolutely continuous mapping satisfying the system

$$
\begin{cases}\left|x^{\prime}(t)\right| \leq p(t)|x(t)| & \text { for a.e. } t \in(a, b) \\ x_{i}\left(t_{i}\right)=0 & \text { for } i=1, \ldots, k\end{cases}
$$

Then $x(t)=0$ for every $t \in[a, b]$.

Let $\|\cdot\|$ be the supremum norm in $C[a, b]$, let $\|\cdot\|_{k}$ be the norm in $C\left([a, b], \mathbb{R}^{k}\right)$ given by $\|x\|_{k}=\sum_{i=1}^{k}\left\|x_{i}\right\|$ for $x=\left(x_{1}, \ldots, x_{k}\right) \in C\left([a, b], \mathbb{R}^{k}\right)$ and let $B(0, r) \subset C\left([a, b], \mathbb{R}^{k}\right)$ be an open ball centred at 0 of radius $r>0$.

Lemma 1. Assume that $F:[a, b] \times \mathbb{R}^{k} \rightarrow \operatorname{cf}\left(\mathbb{R}^{k}\right)$ satisfies all assumptions of Theorem 1. Then:

$$
\begin{aligned}
& \exists_{r>0} \forall_{\lambda \in(0, \beta]} \forall_{\tau \in[0,1]} \quad B(0, r) \cap S_{(\lambda, \tau)}=\{0\} \\
& \forall_{\lambda \geq \alpha} \quad S_{(\lambda, 1)}=\{0\} ; \\
& \forall_{\tau \in[0,1)} \forall_{\lambda \geq \alpha} \quad S_{(\lambda, \tau)}=\emptyset ; \\
& \exists_{K>0} \forall_{\lambda>0} \forall_{x \in C\left([a, b], \mathbb{R}^{k}\right)} \quad x \in S_{(\lambda, 1)} \Rightarrow \sum_{i=3}^{k}\left\|x_{i}\right\| \leq K \\
& \exists_{K>0} \forall_{\lambda \geq 1} \forall_{x \in C\left([a, b], \mathbb{R}^{k}\right)} \quad x \in S_{(\lambda, 1)} \Rightarrow\|x\|_{k} \leq K .
\end{aligned}
$$

Proof of (3.4). By (2.1) there exists $r>0$ such that $|F(t, x)| \leq \psi(t)|x|$ for each $t \in[a, b]$ and $|x| \leq r$. Let $\lambda \in(0, \beta]$ and $x \in B(0, r) \cap S_{(\lambda, 1)}$. Then

$$
\left\{\begin{array}{l}
x^{\prime}(t) \in \tau \lambda F(t, x(t)) \quad \text { for a.e. } t \in(a, b), \\
l(x)=0 .
\end{array}\right.
$$

Hence

$$
\left|x^{\prime}(t)\right| \leq \tau \lambda|F(t, x(t))| \leq \tau \lambda \psi(t)|x(t)| \leq \beta \psi(t)|x(t)| \quad \text { for a.e. } t \in(a, b),
$$

so according to (3.3), $x(t)=0$ for each $t \in[a, b]$.

Proof of (3.5). Let $\lambda \geq \alpha$ and $x \in S_{(\lambda, 1)}$. Then

From the definition of $p$ we obtain

$$
\left\{\begin{array}{l}
x^{\prime}(t)=-\lambda p(x(t)) \\
l(x)=0
\end{array}\right.
$$

so

$$
\left\{\begin{array}{l}
x_{1}^{\prime}(t)=\lambda x_{2}(t) \\
x_{2}^{\prime}(t)=-\lambda M\left|x_{1}(t)\right| \\
x_{1}(a)=0, \quad x_{2}(b)=0
\end{array}\right.
$$

$$
\left\{\begin{array}{l}
x_{1}^{\prime \prime}(t)=-\lambda^{2} M\left|x_{1}(t)\right| \\
x_{1}(a)=0 \\
x_{1}^{\prime}(b)=0
\end{array}\right.
$$


By the maximum principle (cf. [8]), $x_{1}(t) \geq 0$ for every $t \in[a, b]$. Since $\lambda \geq 1$, (3.1) yields $x_{1}(t)=0$ for each $t \in[a, b]$. Therefore $x(t)=0$ for each $t \in[a, b]$, so (3.5) is proved.

Proof of (3.6). Let $x \in S_{(\lambda, \tau)}$ for some $\lambda \geq \alpha$ and $\tau \in[0,1)$. Then

$$
\left\{\begin{array}{l}
x^{\prime}(t)=-\lambda p(x(t))-(1-\tau) x^{0}(t) \\
l(x)=0
\end{array}\right.
$$

hence

$$
\left\{\begin{array}{l}
x_{1}^{\prime \prime}(t)=-\lambda^{2} M\left|x_{1}(t)\right|-\lambda(1-\tau) x_{0}(t) \\
x_{1}(a)=0 \\
x_{1}^{\prime}(b)=0
\end{array}\right.
$$

From (3.2) we conclude that the above problem has no solution. This completes the proof.

Proof of (3.7). Let $x \in S_{(\lambda, 1)}$. By (iii) and (2.3) there exists $m_{R} \in$ $L^{1}(a, b)$ such that

$$
\left|\left(x_{i}\right)^{\prime}(t)\right| \leq m_{R}(t)+\lambda \psi_{i}(t)\left|x_{i}(t)\right| \quad \text { for a.e. } t \in[a, b] \text { and } i=3,4, \ldots, k .
$$

From (3.5) we obtain

$$
\left|\left(x_{i}\right)^{\prime}(t)\right| \leq m_{R}(t)+\alpha \psi_{i}(t)\left|x_{i}(t)\right| \text { for a.e. } t \in[a, b] \text { and } i=3,4, \ldots, k .
$$

Hence by the Gronwall inequality we have

$$
\left|x_{i}(t)\right| \leq \int_{a}^{b} m_{R}(t) e^{\alpha \int_{a}^{b} \psi_{i}(t) d t} d t \quad \text { for } t \in[a, b] \text { and } i=3,4, \ldots, k .
$$

This completes the proof of (3.7).

Proof of (3.8). Suppose that (3.8) is not satisfied, i.e. there exist sequences $\left\{\lambda_{n}\right\} \subset(1, \infty)$ and $\left\{x^{n}\right\} \subset S_{\left(\lambda_{n}, 1\right)}$ such that $\sum_{i=1}^{k}\left\|x_{i}^{n}\right\| \rightarrow \infty$ and $\lambda_{n} \rightarrow \lambda_{0} \geq 1$. From (3.7) we obtain $\left\|x_{1}^{n}\right\|+\left\|x_{2}^{n}\right\| \rightarrow \infty$. Since $x^{n} \in S_{\left(\lambda_{n}, 1\right)}$, we have for a.e. $t \in(a, b)$,

$$
\left\{\begin{array}{l}
\left(x_{1}^{n}\right)^{\prime}(t) \in \lambda_{n} q_{1}\left(\lambda_{n}\right)\left(\Pi_{1} \circ F\right)\left(t, x_{n}(t)\right)+\lambda_{n} q_{2}\left(\lambda_{n}\right) x_{2}^{n}(t), \\
\left(x_{2}^{n}\right)^{\prime}(t) \in \lambda_{n} q_{1}\left(\lambda_{n}\right)\left(\Pi_{2} \circ F\right)\left(t, x_{n}(t)\right)-\lambda_{n} q_{2}\left(\lambda_{n}\right) M\left|x_{1}(t)\right|, \\
x_{1}^{n}(a)=0, \quad x_{2}^{n}(b)=0 .
\end{array}\right.
$$

So there exists a sequence $\left\{w_{i}^{n}\right\} \subset L^{1}(a, b)$ such that $w_{i}^{n}(t) \in\left(\Pi_{i} \circ F\right)\left(t, x^{n}(t)\right)$ a.e. on $[a, b]$ for $i=1,2$ and

$$
\begin{aligned}
& x_{1}^{n}(t)=\lambda_{n} q_{1}\left(\lambda_{n}\right) \int_{a}^{t}\left(w_{1}^{n}(s)-x_{2}^{n}(s)\right) d s+\lambda_{n} \int_{a}^{t} x_{2}^{n}(s) d s, \\
& x_{2}^{n}(t)=\lambda_{n} q_{1}\left(\lambda_{n}\right) \int_{b}^{t}\left(w_{2}^{n}(s)+M\left|x_{1}^{n}(s)\right|\right) d s-\lambda_{n} M \int_{b}^{t}\left|x_{1}^{n}(s)\right| d s .
\end{aligned}
$$


Set $v_{i}^{n}(t)=x_{i}^{n}(t) /\left\|x^{n}\right\|_{k}$. From conditions (iii) and (2.2), and the ArzelàAscoli Theorem, there exists a subsequence of $\left\{v_{i}^{n}\right\}$ which is convergent to $v_{i}$ for $i=1,2$ and the following conditions are satisfied:

$$
\left\{\begin{array}{l}
v_{1}^{\prime}(t)=\lambda_{0} v_{2}(t) \\
v_{2}^{\prime}(t)=-\lambda_{0} M\left|v_{1}(t)\right| \\
v_{1}(a)=0, \quad v_{2}(b)=0
\end{array}\right.
$$

Similarly to what we showed in (3.5), by (3.1) we have $\lambda_{0} M=(\pi / 2(b-a))^{2}$ so necessarily $\lambda_{0}<1$. This contradiction and finishes the proof of (3.8).

Lemma 2. Assume that $F:[a, b] \times \mathbb{R}^{k} \rightarrow \mathrm{cf}\left(\mathbb{R}^{k}\right)$ satisfies all assumptions of Theorem 2. Then there exists $K>0$ such that

$$
\sum_{i=3}^{k}\left\|x_{i}\right\| \leq K \text { for } \lambda \in(0, \infty) \text { and } x \in S_{(\lambda, 1)} .
$$

Proof of (3.9). Suppose (3.9) is not satisfied, i.e. there exist sequences $\left\{\lambda_{n}\right\}$ and $\left\{x^{n}\right\} \subset S_{\left(\lambda_{n}, 1\right)}$ such that $\sum_{i=3}^{k}\left\|x_{i}^{n}\right\| \rightarrow \infty$ and $\lambda_{n} \rightarrow \lambda_{0}<\alpha$. Set $y_{n}=\left(x_{3}^{n}, \ldots, x_{k}^{n}\right)$. By (iii) and (2.4) there exists $m_{R} \in L^{1}(a, b)$ such that

$$
\left\{\begin{array}{l}
\left|y_{n}^{\prime}(t)\right| \leq m_{R}(t)+\lambda_{n} \psi(t)\left|y_{n}(t)\right| \text { a.e. } t \in[a, b], \\
l\left(y_{n}\right)=0 .
\end{array}\right.
$$

Observe that the function $v_{n}=y_{n} /\left\|y_{n}\right\|_{k-2}$ is a solution of the problem

$$
\left\{\begin{array}{l}
\left|v_{n}^{\prime}(t)\right| \leq \frac{m_{R}(t)}{\left\|y_{n}\right\|_{k-2}}+\lambda_{n} \psi(t)\left|v_{n}(t)\right| \quad \text { a.e. } t \in[a, b], \\
l\left(v_{n}\right)=0 .
\end{array}\right.
$$

Therefore the sequence $\left\{v_{n}^{\prime}\right\}$ of derivatives is bounded by the integrable function $\alpha \psi_{1}+m_{R}$ for $n \in \mathbb{N}$ large enough. Then by the Pliś Lemma [7] there exists a subsequence $\left\{v_{n_{k}}\right\}$ of $\left\{v_{n}\right\}$ uniformly convergent to an absolutely continuous function $v_{0}:[a, b] \rightarrow \mathbb{R}^{k-2}$ which is a non-trivial solution of the problem (3.3). This contradiction finishes the proof of (3.9).

4. Proofs of Theorems 1 and 2. To the Carathéodory mapping $F:[a, b] \times \mathbb{R}^{k} \rightarrow \mathrm{cf}\left(\mathbb{R}^{k}\right)$ we associate the Nemytskiu operator $\mathcal{F}: C\left([a, b], \mathbb{R}^{k}\right)$ $\rightarrow \operatorname{cf}\left(L^{1}\left((a, b), \mathbb{R}^{k}\right)\right)$, given by

$$
\mathcal{F}(x)=\left\{w \in L^{1}\left((a, b), \mathbb{R}^{k}\right): w(t) \in F(t, x(t)) \text { for a.e. } t \in(a, b)\right\} .
$$

Let $P: C\left([a, b], \mathbb{R}^{k}\right) \rightarrow L^{1}\left((a, b), \mathbb{R}^{k}\right)$ be the Nemytskir operator for the mapping $p: \mathbb{R}^{k} \rightarrow \mathbb{R}^{k}$, and let $T=\left(T_{1}, \ldots, T_{k}\right): L^{1}\left((a, b), \mathbb{R}^{k}\right) \rightarrow C\left([a, b], \mathbb{R}^{k}\right)$ be the integral operator given by

$$
T(x)=\left(T_{1}\left(x_{1}\right), \ldots, T_{k}\left(x_{k}\right)\right) \quad \text { where } \quad T_{i}\left(x_{i}\right)(t)=\int_{t_{i}}^{t} x_{i}(s) d s .
$$


With the family of boundary value problems $\left(2_{(\lambda, \tau)}\right)$ we associate the family of vector fields $f_{\tau}:(0, \infty) \times C\left([a, b], \mathbb{R}^{k}\right) \rightarrow \operatorname{cf}\left(C\left([a, b], \mathbb{R}^{k}\right)\right)$ given by

$$
f_{\tau}(\lambda, x)=x-\lambda \tau q_{1}(\lambda) T \mathcal{F}(x)+\lambda q_{2}(\lambda) T P(x)+(1-\tau) q_{2}(\lambda) T\left(x^{0}\right) .
$$

Observe that $x \in S_{(\lambda, \tau)}$ iff $0 \in f_{\tau}(\lambda, \tau)$. Moreover the vector field $f_{\tau}$ is completely continuous (cf. [9], [10]).

We call $(\mu, 0) \in(0, \infty) \times C\left([a, b], \mathbb{R}^{k}\right)$ a bifurcation point of the mapping $f_{1}$ if for every open subset $U \subset(0, \infty) \times C\left([a, b], \mathbb{R}^{k}\right)$ with $(\mu, 0) \in U$ there exists a point $(\lambda, x) \in U$ such that $x \neq 0$ and $0 \in f_{1}(\lambda, x)$. Denote by $\mathcal{B}_{f_{1}}$ the set of all bifurcation points of $f_{1}$. Let $\mathcal{R}_{f_{1}} \subset(0, \infty) \times C\left([a, b], \mathbb{R}^{k}\right)$ be the closure (in $\left.(0, \infty) \times C^{1}\left([a, b], \mathbb{R}^{k}\right)\right)$ of the set of non-trivial solutions of the inclusion $0 \in f_{1}(\lambda, x)$, i.e.

$$
\mathcal{R}_{f_{1}}=\overline{\left\{(\lambda, x) \in(0, \infty) \times C\left([a, b], \mathbb{R}^{k}\right): x \neq 0 \wedge 0 \in f_{1}(\lambda, x)\right\}} .
$$

For each $\lambda$ satisfying $(\lambda, 0) \notin \mathcal{B}_{f_{1}}$ there exists $r_{0}>0$ such that $0 \notin f_{1}(\lambda, x)$ for $\|u\|=r \in\left(0, r_{0}\right]$, so the value $\operatorname{deg}\left(f_{1}(\lambda, \cdot), B(0, r), 0\right)$ is defined.

Assume that for an interval $[c, d] \subset(0, \infty)$ there exists $\delta>0$ such that

$$
(([c-\delta, c) \cup(d, d+\delta]) \times\{0\}) \cap \mathcal{B}_{f_{1}}=\emptyset .
$$

Then we may define the bifurcation index $s\left[f_{1}, c, d\right]$ of the mapping $f_{1}$ with respect to the interval $[c, d]$ as

$$
s\left[f_{1}, c, d\right]=\lim _{\lambda \rightarrow d^{+}} \operatorname{deg}\left(f_{1}(\lambda, \cdot), B(0, r), 0\right)-\lim _{\lambda \rightarrow c^{-}} \operatorname{deg}\left(f_{1}(\lambda, \cdot), B(0, r), 0\right),
$$

where $r=r(\lambda)>0$ is small enough.

The main tool used in this section, Theorem A below, is a global bifurcation theorem for convex-valued completely continuous mappings which is a consequence of a generalization of the Rabinovitz global bifurcation alternative (see [1], [11]).

Theorem A (see [3]). Let $f_{1}:(0, \infty) \times C\left([a, b], \mathbb{R}^{k}\right) \rightarrow \operatorname{cf}\left(C\left([a, b], \mathbb{R}^{k}\right)\right)$ be given by $\left(4_{1}\right)$, and assume that there exists an interval $[c, d] \subset(0, \infty)$ such that $\mathcal{B}_{f_{1}} \subset[c, d] \times\{0\}$ and $s\left[f_{1}, c, d\right] \neq 0$. Then there exists a non-compact component $\mathcal{C} \subset \mathcal{R}_{f_{1}}$ satisfying $\mathcal{C} \cap \mathcal{B}_{f_{1}} \neq \emptyset$.

Proof of Theorem 1. According to (3.4) and (3.5), $\mathcal{B}_{f_{1}} \subset[\beta, \alpha]$. Observe that by (3.4) for $\lambda<\beta$ there exists $r>0$ such that $f_{1}(\lambda, \cdot): B(0, r) \rightarrow$ $\operatorname{cf}\left(C[a, b], \mathbb{R}^{k}\right)$ is homotopic to the identity mapping. Hence by the homotopy property of the topological degree we have $\operatorname{deg}\left(f_{1}(\lambda, \cdot), B(0, r), 0\right)=1$. According to (3.5) and (3.6), for $\lambda>\alpha$ there exists $r>0$ such that $f_{1}(\lambda, \cdot): B(0, r) \rightarrow \operatorname{cf}\left(C[a, b], \mathbb{R}^{k}\right)$ is homotopic to the mapping $f_{0}(\lambda, \cdot)$ which has no zeros and $\operatorname{deg}\left(f_{1}(\lambda, \cdot), B(0, r), 0\right)=0$. Therefore $s\left[f_{1}, \beta, \alpha\right]=-1$. According to Theorem A there exists a non-compact component $\mathcal{C} \subset \mathcal{R}_{f_{1}}$ satisfying $\mathcal{C} \cap \mathcal{B}_{f_{1}} \neq \emptyset$. Since $\mathcal{C}$ is not compact there exists a sequence $\left\{\left(\lambda_{n}, x_{n}\right)\right\} \subset \mathcal{C}$ such that $\left\|x_{n}\right\|_{k} \rightarrow \infty$, or $\lambda_{n} \rightarrow \infty$, or $\lambda_{n} \rightarrow 0$. Observe that 
by (3.5) the case $\lambda_{n} \rightarrow \infty$ is impossible. Now consider the case $\left\|x_{n}\right\|_{k} \rightarrow \infty$. Then it follows from (3.8) that $\lambda_{n}<1$ for $n \in \mathbb{N}$ large enough. So the connected set $\mathcal{C}$ contains pairs $\left(\lambda_{1}, x_{1}\right)$ and $\left(\lambda_{2}, x_{2}\right)$ with $\lambda_{1}<1$ and $\lambda_{2}>1$. Hence there exists $(1, x) \in \mathcal{C}$. This solution of the inclusion $0 \in f_{1}(1, x)$ must have $x \neq 0$ because $(1,0) \notin \mathcal{R}_{f_{1}}$. The proof is complete.

Proof of Theorem 2. Since $F$ satisfies assumptions (2.1) and (2.2) of Theorem 1, there exists a non-compact component $\mathcal{C} \subset \mathcal{R}_{f_{1}}$ satisfying $\mathcal{C} \cap$ $\mathcal{B}_{f_{1}} \neq \emptyset$. Similarly to what we showed in the proof of Theorem 1, from (3.9) and (3.8) it follows that there exist $\lambda_{1}<1$ and $x \in C\left([a, b], \mathbb{R}^{k}\right)$ such that $\left(\lambda_{1}, x\right) \in \mathcal{C}$. Because $\mathcal{B}_{f_{1}} \subset[\beta, \alpha]$ we can see that the connected set $\mathcal{C}$ contains pairs $\left(\lambda_{1}, x_{1}\right)$ and $\left(\lambda_{2}, x_{2}\right)$ with $\lambda_{1}<1$ and $\lambda_{2}>1$. Hence $(1, x) \in \mathcal{C}$ for some $x \neq 0$ as before. The proof is complete.

Acknowledgements. The author is grateful to Professor Tadeusz Pruszko for the inspiration and help during the preparation of this article.

\section{References}

[1] N.-S. Chow and J. K. Hale, Methods of Bifurcation Theory, Springer, 1982.

[2] E. Coddington and N. Levinson, Theory of Ordinary Differential Equations, McGraw-Hill, New York, 1955.

[3] S. Domachowski and J. Gulgowski, A global bifurcation theorem for convex-valued differential inclusions, Z. Anal. Anwendungen 23 (2004), 275-292.

[4] P. Hartman, Ordinary Differential Equations, Birkhäuser, Boston, 1982.

[5] A. Lasota and C. Olech, An optimal solution of Nicoletti's boundary value problem, Ann. Polon. Math. 18 (1966), 131-139.

[6] A. Lasota and Z. Opial, Fixed-point theorems for multi-valued mappings and optimal control problems, Bull. Acad. Polon. Sci. 16 (1968), 645-649.

[7] A. Pliś, Measurable orientor fields, ibid. 13 (1965), 565-569.

[8] M. H. Protter and H. F.Weinberger, Maximum Principles in Differential Equations, Springer, New York, 1984

[9] T. Pruszko, Topological degree methods in multi-valued boundary value problems, Nonlinear Anal. 5 (1981), 959-973.

[10] - Some applications of the topological degree theory to multi-valued boundary value problems, Dissertationes Math. 229 (1984).

[11] P. Rabinowitz, Some global results for nonlinear eigenvalue problems, J. Funct. Anal. 7 (1971), 487-513.

Institute of Mathematics

University of Gdańsk

Wita Stwosza 57

80-952 Gdańsk, Poland

E-mail: mdom@math.univ.gda.pl 